\title{
PERFORMANCE EVALUATION OF ATENOLOL MOLECULAR IMPRINTED POLYMER USING TWO DIFFERENT POLYMERIZATION AND TWO DIFFERENT POROGEN
}

\author{
A. N. Hasanah*, M. Suherman, I. Susanti, I. Pitaloka and R. Mustarichie \\ Pharmaceutical Analysis and Medicinal Chemistry Department, Faculty of Pharmacy, \\ Universitas Padjadjaran \\ *E-mail: aliya.n.hasanah@unpad.ac.id
}

\begin{abstract}
Performance characterization four types of Molecular Imprinted Polymer (MIP) atenolol has been carried out with two different types of porogens(butanol and propanol) and polymerization methods (bulk and precipitation). Four polymers were optimized and applied to the serum, results showed that the polymer made by the precipitation method in butanol solvents produced the best recovery value (74.64\%) and have mesopore form based on pore size. Meanwhile, the results of physical characterization showed that there was no functional group change between sorbent before and after applied as SPE cartridges. Sorbent made by bulk method had better physical stability than sorbent made by the precipitation method.

Keywords: MIP Performance Evaluation, Atenolol Molecular Imprinted Polymer, Butanol Porogen, Propanol Porogen, Polymer Imprinting Factor
\end{abstract}

(C) RASĀYAN. All rights reserved

\section{INTRODUCTION}

Atenolol is a beta-blocker class of drugs that are used singly or in combination for the treatment of hypertension, angina pectoris, arrhythmias, and myocardial infarction ${ }^{1}$. However, atenolol is also considered as doping for athletes because it has effects that can reduce systolic and diastolic blood pressure $^{2}$. To date, the atenolol testing method is nano-liquid mass chromatography-mass spectrometry ${ }^{3}$, differential voltage voltammetry method ${ }^{4}, \mathrm{AAS}^{5}$, high-performance thin-layer chromatography ${ }^{6}$, and reverse-phase liquid chromatography with UV detectors. ${ }^{7}$ The methods used for the analysis of atenolol have a weakness regarding high costs to get good sensitivity. Therefore, a specific preparation method needs to be developed so that the analysis of atenolol can be carried out with UV detector HPLC instrumentation that is common in testing laboratories. Nowadays, sample preparation is developed using specific Solid Phase Extraction (SPE), which is through the formation of Molecularly Imprinted Polymer (MIP). By using this preparation method, it is expected to reduce some of the weaknesses above. This MIP preparation method allows the low detection of analytes that can be carried out using the HPLC with a common detector found in every institution.

Molecularly Imprinted Polymer (MIP) is a synthetic material designed to have selectivity for predetermined molecular targets and can be synthesized by self-assembly through a non-covalent or covalent complex pre-polymerization bond between molecules and functional monomers in suitable porogen. The pre-polymerization complex is then polymerized with the help of a crosslinker after the template is taken from the polymer formed. Template retrieval can be done with a simple extraction procedure, or chemical termination, depending on the type of interaction between the template and the monomer. Polymers will then have unique recognition sites, complementary shapes, sizes, and functions, to template molecules, or structural analogs that have similar structures. ${ }^{4}$ The functional monomer of methacrylic acid (MAA) is a universal monomer and is very often used based on its potential, MAA is

Rasayan J. Chem., 12(3), 1269-1278(2019)

http://dx.doi.org/10.31788/RJC.2019.1235202

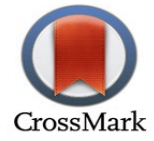


known as a versatile monomer for molecular imprinting and shows dimerization reactions that enhance the imprinting effect ${ }^{8}$. Synthesis of MI-SPE atenolol-based on non-covalent bonds using MAA functional monomers has produced suitable sorbent. ${ }^{9-12}$

There are two methods to make MIP, namely bulk and precipitation method. The bulk method is a method that is widely used in the synthesis of MIP because it is fast, simple, and does not require particular expertise or sophisticated instruments in its manufacture ${ }^{4}$. The product produced requires grinding and filtrating before use. The grinding process, besides spending much time can also damage some bonding sites and reduce molecular recognition ability and selectivity of polymers obtained from the deposition polymerization method, which is another alternative in the manufacture of $\mathrm{MIP}^{4}$. The precipitation method uses a mixing reaction with the bulk method, except for the use of more porogen solvents. Uniform particles can be obtained using this method, where the process of forming polymer chains continues to grow until they are more significant to become insoluble in the mixed reaction.

Furthermore, granular polymer particles are easily obtained by washing and centrifugation. This technique is easy and requires a shorter time than the bulk method and produces regular granules in large quantities ${ }^{13}$. This research was done to evaluate two different polymerization method using methacrylic acid as a functional monomer in order to know their performance as a sample preparation method for the recognition of atenolol in serum samples.

\section{Materials}

\section{EXPERIMENTAL}

High Performance Liquid Chromatography (HPLC) (Dionex Ultimate 3000), BET surface area analyzer (NOVA instruments (c) 1994-2010, Quantachrome Instruments version 11.0), Scanning Electron Microscope with Energy Dispersive Spectroscopy (SEM / EDS) (JEOL JSM-6360 LA), FourierTransform Infrared Spectroscopy (FTIR) (prestige-21 Shimadzu), 250x4,0 mm Column ( LiChrospher ${ }^{\circledR}$ 100 RP-18 (Merck), SPE Cartridge (Supelco column SPE superclean LC-Si), oven (Memmert), UVspectrophotometer Vis (Analytik Jenaspecord 200), digital scales (Ohaus Pioneer), ultrasonic (NEY 19H), water bath (Memmert), and glassware commonly used in laboratories.

Atenolol (TCI), acetonitrile HPLC gradient grade (JT Baker and Fisher Scientific), methanol HPLC gradient grade (JT Baker and Fisher Scientific), trifluoroacetic acid (Sigma Aldrich), triethylamine (Sigma Aldrich) and glacial acetic acid (JT Baker and Fisher Scientific). Methacrylate acid (Aldrich), Ethylene glycol dimethacrylate (Aldrich), Benzoyl peroxide (TCI), butanol (Merck), Propranol (Merck). All reagents used have an analysis grade unless stated otherwise.

\section{Methods}

\section{Monomer-Template Association Constant Determination}

Determination of the constant association of template monomers was carried out using UV-Vis spectrophotometry. Atenolol $2 \times 10^{-5} \mathrm{M}$ solution in propanol and butanol was measured by UV spectrophotometer. Then, $5 \times 10^{-3} \mathrm{M}$ methacrylic acid is added little by little. Each addition of methacrylic acid is measured for absorption, and then the association constants are calculated using the BenesiHildebran equation:

$$
\frac{1}{\Delta Y}=\frac{1}{Y \Delta \mathrm{HGKa}[\mathrm{G}]}+\frac{1}{Y \Delta \mathrm{HG}}
$$

Where, $\Delta \mathrm{Y}$ is a change in absorbance, $\mathrm{Y} \Delta \mathrm{HG}$ is a change in absorbance with absorbance at the end of the titration, $\mathrm{Ka}$ is an association constant, and $[\mathrm{G}]$ is an added monomer concentration. ${ }^{14}$

\section{Synthesis of MIP with Bulk Polymerization Method}

Atenolol (template) 0.2663 grams was dissolved in $10 \mathrm{~mL}$ of propanol and butanol in a closed vial and sonicated for 5 minutes until atenolol dissolved. Monomers of methacrylic acid (functional monomers) of $340 \mu \mathrm{L}$ were added and sonicated for 20 minutes. EGDMA (crosslinker) $3.77 \mathrm{~mL}$ was added and sonicated for 5 minutes. Benzoyl peroxide as an initiator as much as $250 \mathrm{mg}$ is added and sonicated for 20 
minutes (until dissolved). The vial was sealed using parafilm and heated in the oven for 1 hour at $70{ }^{\circ} \mathrm{C}$. The vial was then transferred to a waterbath at $70^{\circ} \mathrm{C}$ for 18 hours. The formed polymer is crushed and sieved with a 60 mesh. The polymer is washed using $20 \mathrm{~mL}$ of methanol, then is dried in an oven at $50^{\circ}$ C. The polymer then was extracted using soxhlet for 24 hours with methanol: acetic acid (9: 1). After extracting with Soxhlet, sorbent was dried in the oven for 18 hours at $50{ }^{\circ} \mathrm{C}$. Monitoring with a UV spectrophotometer was carried out to ensure that atenolol was fully extracted. In addition to MIP, NonImprinted Polymers (NIP) were made with the same procedure without the addition of atenolol (template).

\section{Synthesis of MIP-SPE Polymers by Precipitation Polymerization Method}

Atenolol (template) 0.2663 grams was dissolved in $50 \mathrm{~mL}$ of propanol and butanol in a closed glass bottle and sonicated until atenolol dissolved. $340 \mu \mathrm{L}$ of methacrylic acid (functional monomers) and $300 \mathrm{~mL}$ of propanol/butanol were added and sonicated for 20 minutes. EGDMA (crosslinker) $3.77 \mathrm{~mL}$ was added and sonicated for 5 minutes. Benzoyl peroxide as an initiator as much as $250 \mathrm{mg}$ is added and sonicated for 20 minutes (until dissolved). The bottle is sealed using parafilm and heated in the oven for 1 hour at $70{ }^{\circ} \mathrm{C}$. The vial was then transferred into a shaker waterbath at $70^{\circ} \mathrm{C}$ for 18 hours. The polymer was filtered and washed using $40 \mathrm{~mL}$ methanol, after which the polymer was dried in an oven at $50^{\circ} \mathrm{C}$. The polymer is then extracted with the same procedure as the bulk method. Besides MIP, Non-Imprinted Polymers (NIP) were made with the same procedure without the addition of atenolol (template).

\section{Adsorption Capacity Evaluation of MIP and NIP}

The concentration of atenolol solution is $2.5 ; 5 ; 7.5 ; 10,12.5$ and $15 \mathrm{ppm}$ are prepared. $5 \mathrm{ml}$ of atenolol solution 7.5; 10 and $12.5 \mathrm{ppm}$ were added to the vial containing $20 \mathrm{mg}$ MIP sorbent in the bulk method and $2.5 ; 10,15 \mathrm{ppm}$ was put into a vial containing $20 \mathrm{mg}$ sorbent MIP precipitation method. Shaking the solution for 5 minutes then was done and left it for 24 hours. The mixture was then decanted,and the absorbance of the filtrate was measured using a UV spectrophotometer. The amount of atenolol absorbed was calculated based on the difference in the initial concentration of atenolol and the final concentration of atenolol in the filtrate. This evaluation is also carried out on the NIP with the same method.

\section{Selectivity Determination of MIP and NIP}

Determination of selectivity was carried out by adding atenolol analog solutions including metoprolol and propranolol. $5 \mathrm{~mL}$ of a $5 \mathrm{ppm}$ concentration solution from each substance solution was put into a different vial which contained $20 \mathrm{mg}$ of MIP sorbent then shaking for 5 minutes and allowed to stand for 24 hours. The mixture was then decanted,and the absorbance of the filtrate was measured using a UV spectrophotometer.

\section{MI-SPE Application of Atenolol Extraction in Serum Samples}

The application of MI-SPE method was based on our research with the same HPLC condition and sample preparation ${ }^{15}$.

\section{Physical Characterization}

Polymer Physical Characterization testing was carried out by FTIR, SEM, and Surface Area Analysis to obtain BET adsorption isotherm curves.

\section{Monomer-Template Association Constant Determination}

\section{RESULTS AND DISCUSSION}

Based on the curve in Fig.-1, the Ka value is $924 \mathrm{M}^{-1}$, this value is still less than expected, $10^{3} \mathrm{M}^{-1}$, but approaching that value defines the strength of the interaction that occurs, this Ka value will determine the selectivity and specificity of the polymer produced ${ }^{16}$.

Based on the Benesi-Hildebrand equation, the association constant between atenolol and methacrylic acid in propanol solvents (Fig.-2) is $5.1 \times 10^{2} \mathrm{M}$. 
RASĀYAN J. Chem.

Vol. 12 | No. 3 |1269 - 1278| July - September | 2019

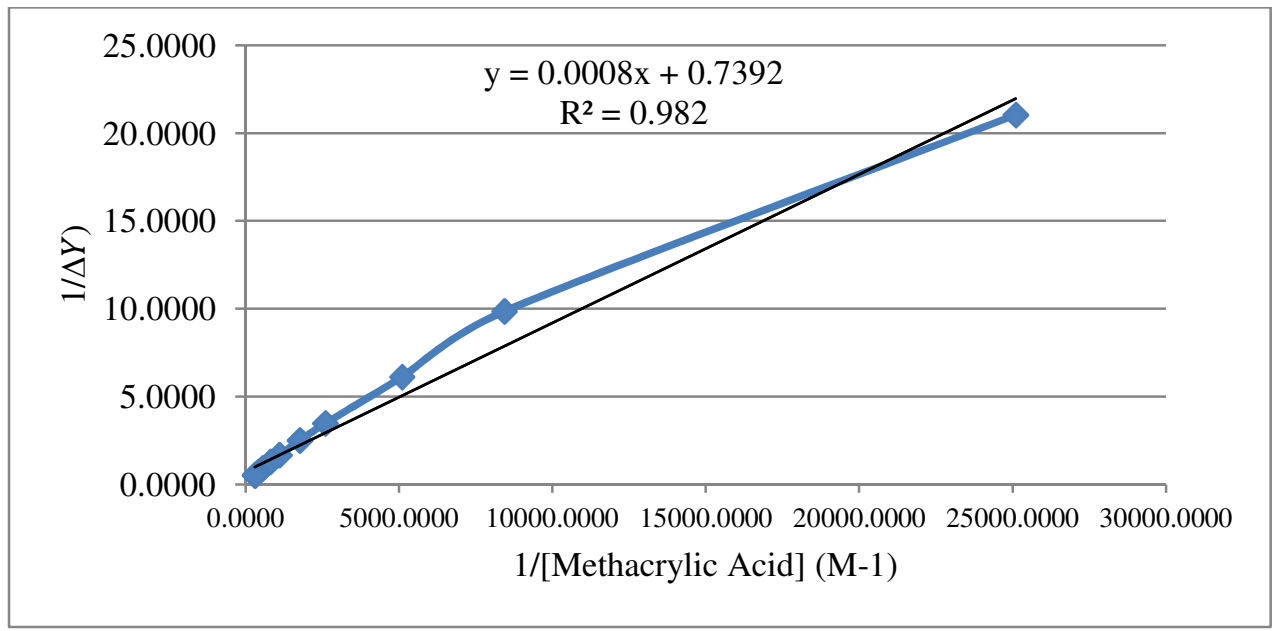

Fig.-1: Association Constant Determination of Atenolol-Methacrylic Acid in Butanol

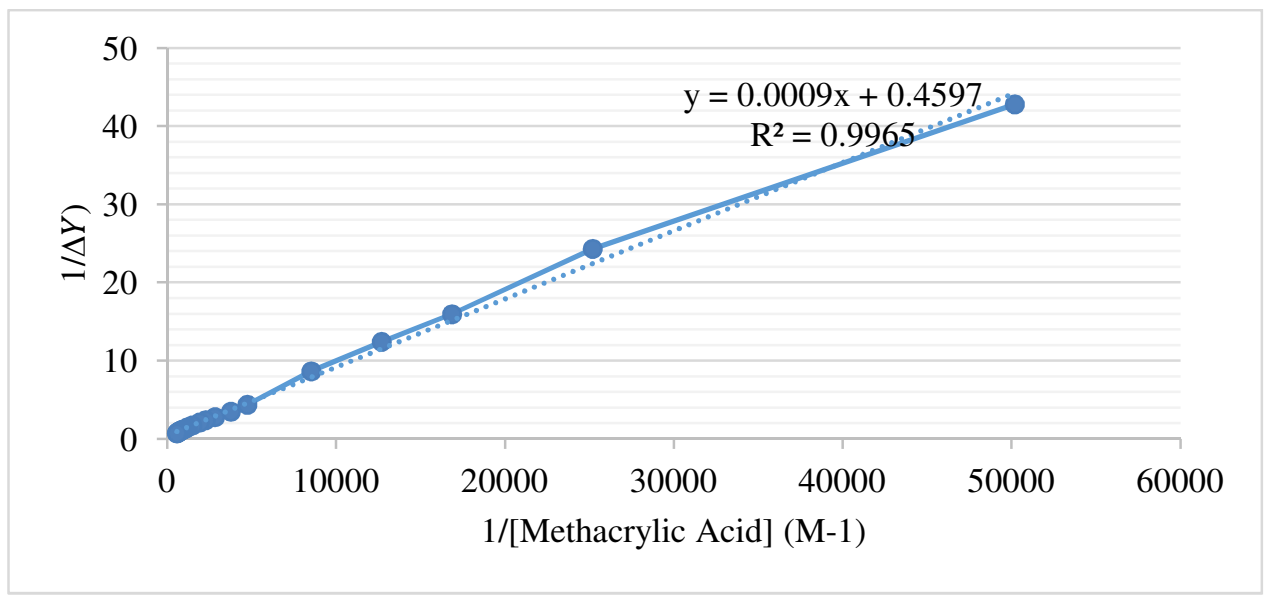

Fig.-2: Association Constant Determination of Atenolol-Methacrylic Acid in Propanol

\section{Molecular Imprinted Polymer Synthesis}

MAA was chosen as a functional monomer because it can form hydrogen bonds with the template atenolol. The carboxylic group of methacrylic acid can bind to the template (atenolol) to form hydrogen bonds such as $\mathrm{O}-\mathrm{H}$ and or with $\mathrm{N}-\mathrm{H}^{17}$. After that, EGDMA (crosslinker) is added and sonicated for 5 minutes. EGDMA (ethylene glycol dimethacrylate) acts as a template that can play a role in controlling morphology and stabilizing the binding area of the resulting polymer ${ }^{18}$. Benzoyl peroxide (BPO) as an initiator is added and sonicated for $20 \mathrm{~min}$ (until dissolved). The purpose of sonication is to remove oxygen in solutions that can inhibit the polymerization process ${ }^{19}$. The container is sealed using parafilm and heated in the oven for 1 hour at $70^{\circ} \mathrm{C}$. The container is then transferred to a waterbath at $70^{\circ} \mathrm{C}$ for 18 hours. For the precipitation method, the shaker on the waterbath is turned on. The thermal decomposition of BPO initiates free radicals polymerization ${ }^{17}$.

\section{Adsorption Capacity Evaluation of MIP and NIP}

Table-1 shows that the distribution of binding sites in MIP and NIP methods is more homogeneous because the value of $\mathrm{m}$ is closer to $1^{20}$. As seen in Table-1, the MIP from precipitation polymerization method has an affinity value for atenolol which is smaller than the Bulk method. From Table-1, the affinity of the NIP from a bulk method has a higher value than the MIP; this is probably due to greater NIP heterogeneity so that the adsorption ability of each side in the sorbent is different. 
RASĀYAN J. Chem.

Vol. 12 | No. 3 |1269 - 1278| July - September | 2019

Table-1: Freundlich Parameter of MIP and NIP in Propanol as Porogenic Solvent

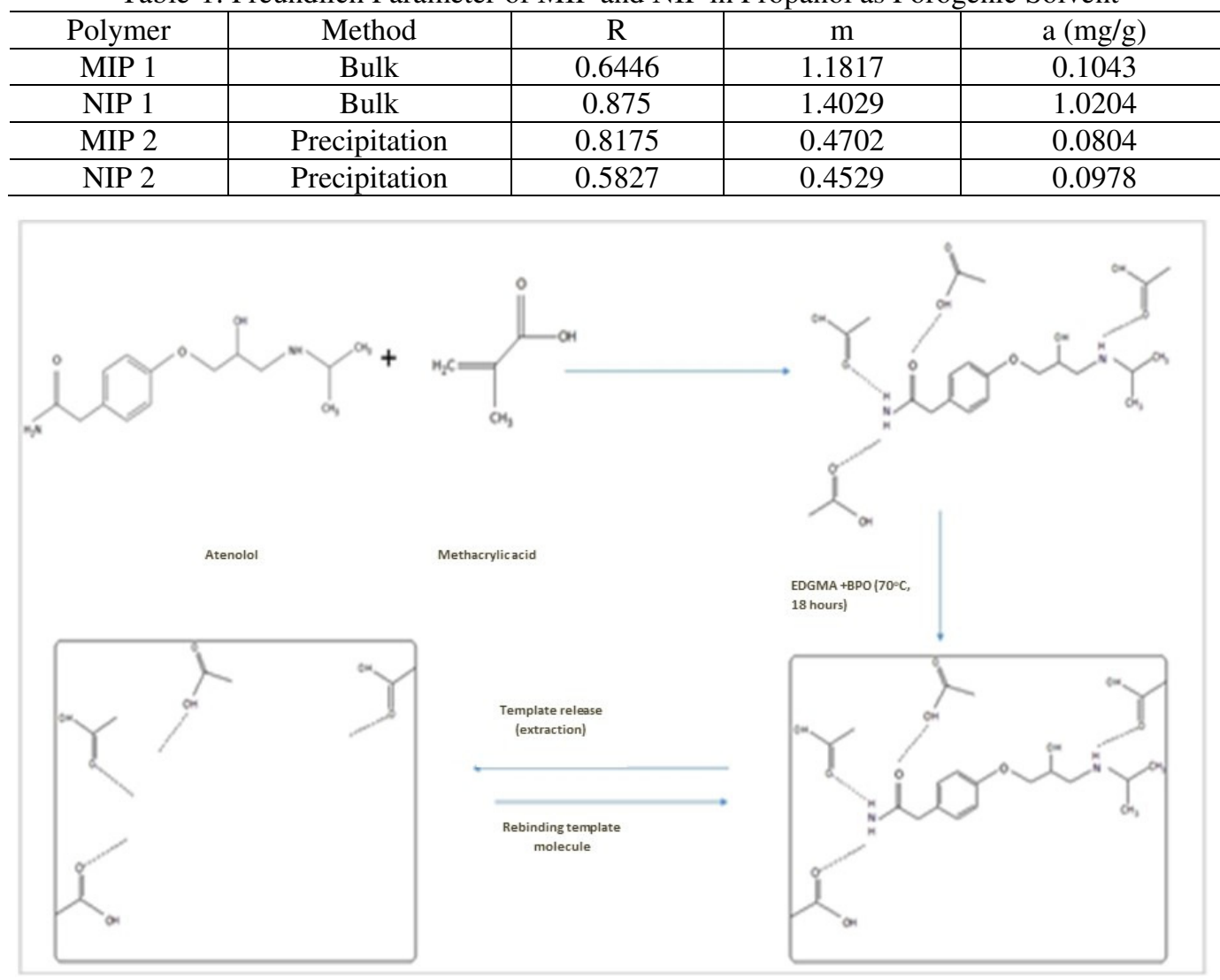

Fig.-3: Synthesis Illustration Scheme of MIP Atenolol-methacrylic Acid Monomer

Table-2: Freundlich Parameter of MIP and NIP in Butanol as Porogenic Solvent

\begin{tabular}{c|c|c|c|c}
\hline Polymer & Method & $\mathrm{R}$ & $\mathrm{m}$ & $\mathrm{a}(\mathrm{mg} / \mathrm{g})$ \\
\hline MIP 3 & Bulk & 0.808 & 0.893 & 7.804 \\
\hline NIP 3 & Bulk & 0.993 & 0.757 & 4.819 \\
\hline MIP 4 & Precipitation & 0.825 & 2.567 & 2.950 \\
\hline NIP 4 & Precipitation & 0.706 & 1.197 & 5.740 \\
\hline
\end{tabular}

Sorbent obtained from bulk polymerization method (MIP 3) in butanol has m values of 0.893 for MIP and 0.757 for NIP, MIP values are closer to 1 , meaning MIP is more homogeneous than NIP. Value describes the capacity of sorbent in absorbing the analyte, MIP can absorb up to $7.804 \mathrm{mg} / \mathrm{gram}$, better than NIP, which is $4.819 \mathrm{mg} / \mathrm{gram}$; this illustrates the strong effect of imprinting. However, this value is still relatively small because a small amount of analyte can be absorbed in the sorbent, so it takes a large amount of sorbent to absorb more analytes. Sorbents synthesized by the precipitation method in butanol have capacities of $2.95 \mathrm{mg} / \mathrm{g}$ for MIP and $5.74 \mathrm{mg} / \mathrm{g}$ for NIP. This value indicates that MIP is less superior than NIP. The NIP homogeneity value is 1.459 (closer to 1) compared to the MIP value of 2.512, which means that the NIP is more homogeneous than MIP.

Based on this test, the MIP adsorption capacity of the bulk method is better than the precipitation method. Research evidence shows that better particle-sized that usually obtained from precipitation method are not always provide a better result. This is because the total pore volume of the MIP from precipitation method maybe lower, and particle porosity is extreme, affecting the performance of extraction ${ }^{21}$.

\section{Selectivity Determination of MIP and NIP}

In this study, as be seen in Table-3, MIP sorbent synthesized by bulk polymerization method (MIP 3) has a high KD value at atenolol which is $486.969 \mathrm{ml} / \mathrm{g}$, this value is much higher than the value of KD 
metoprolol $195.044 \mathrm{ml} / \mathrm{g}$, and propranolol 101.421. Likewise, in precipitation MIP (MIP 4), KD atenolol is higher than metoprolol $207.616 \mathrm{ml} / \mathrm{g}$ and propranolol. This can be interpreted that sorbent MIP is quite selective for atenolol because the KD value is always more significant than the others.

Table-3: Sorbent Selectivity of MIP and NIP in Butanol as Porogenic Solvent

\begin{tabular}{c|c|c|c}
\hline Analyte & Atenolol & Propranolol & Metoprolol \\
\hline KD MIP 3 & 486,969 & 101,421 & 195,044 \\
\hline KD NIP 3 & 169,796 & 83,186 & 530,728 \\
\hline Imprinting Factor & 2,868 & 1,219 & 0,368 \\
\hline KD MIP 4 & 325,435 & 44,508 & 207,616 \\
\hline KD NIP 4 & 78,223 & 68,790 & 191,297 \\
\hline Imprinting Factor & 4,160 & 0,647 & 1,085 \\
\hline
\end{tabular}

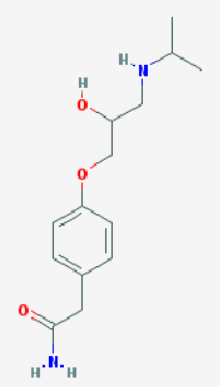

Fig.-4: Atenolol Structu

MIP is synthesized with atenolol as a template molecule so in order to increase the selectivity to atenolol. Based on Figure 4., although all three beta-blockers have hydroxylamine groups, in propranolol, there are two aromatic rings. Structure of metoprolol is more similar to atenolol, which has one aromatic ring, which binds to methanamide or secondary amines to atenolol, and dimethoxyethane for metoprolol.

Atenolol IF values obtained from both bulk and precipitation methods in butanol solvent are higher than one (1) which means that MIP performance is better than NIP and greater than IF metoprolol or propranolol. Based on the selectivity test, the MIP synthesized from precipitation method was superior to the bulk method, but both had good performance compared to NIP and were selective for atenolol.

Table 4. Sorbent Selectivity of MIP and NIP in Propanol as Porogenic Solvent

\begin{tabular}{c|c|c|c}
\hline Analyte & Atenolol & Propranolol & Metoprolol \\
\hline KD MIP 1 & 500.87 & 62.76 & 79.95 \\
\hline KD NIP 1 & 174.42 & 132.40 & 89.21 \\
\hline Imprinting Factor & 2.872 & 0.474 & 0.896 \\
\hline KD MIP 2 & 494.48 & 135.21 & 75.62 \\
\hline KD NIP 2 & 42.19 & 829.27 & 53.03 \\
\hline Imprinting Factor & 11.721 & 0.163 & 1.535 \\
\hline
\end{tabular}

From Table-4, the imprinting factors of MIP from both polymerization method in propanol as porogenic solvents is selective for atenolol. The resulting IF value also shows that IF metoprolol is lower than atenolol and higher than propranolol.

\section{MI-SPE Application of Atenolol Extraction in Serum Samples}

Application of separation of serum samples using MIP 2 obtained recovery value of $10.86 \%$; NIP 2 yielded a value of $47.83 \%$ while MIP 1 was $66.54 \%$ and NIP $135.47 \%$. The percent recovery value is 
obtained through twice elution. Based on the results of percent recovery, it is known that bulk provides a better percent recovery value compared to precipitation MIP, but both do not meet the requirements for recovery values for biological samples so sorbents can not be used as atenolol extracting material from serum samples ${ }^{23}$.

The application of separation of serum samples using MIP 4 obtained a percent recovery value of 74.64\%; NIP 4 gave the results of the percent recovery value of $38.84 \%$ while MIP 3 was at $32.22 \%$ and NIP 3 is $42.31 \%$. Based on the results of the percent recovery value, it was found that MIP 4 gave a better percent recovery value compared to MIP 3 and there was a significant difference between MIP 4 and NIP 4; this was due to differences in polymer size made by precipitation and bulk method. The polymers made by the precipitation method have a smaller particle size, which causes more surface area to interact with the analyte so that the polymer has a higher percent recovery value than the polymer made with the bulk method.

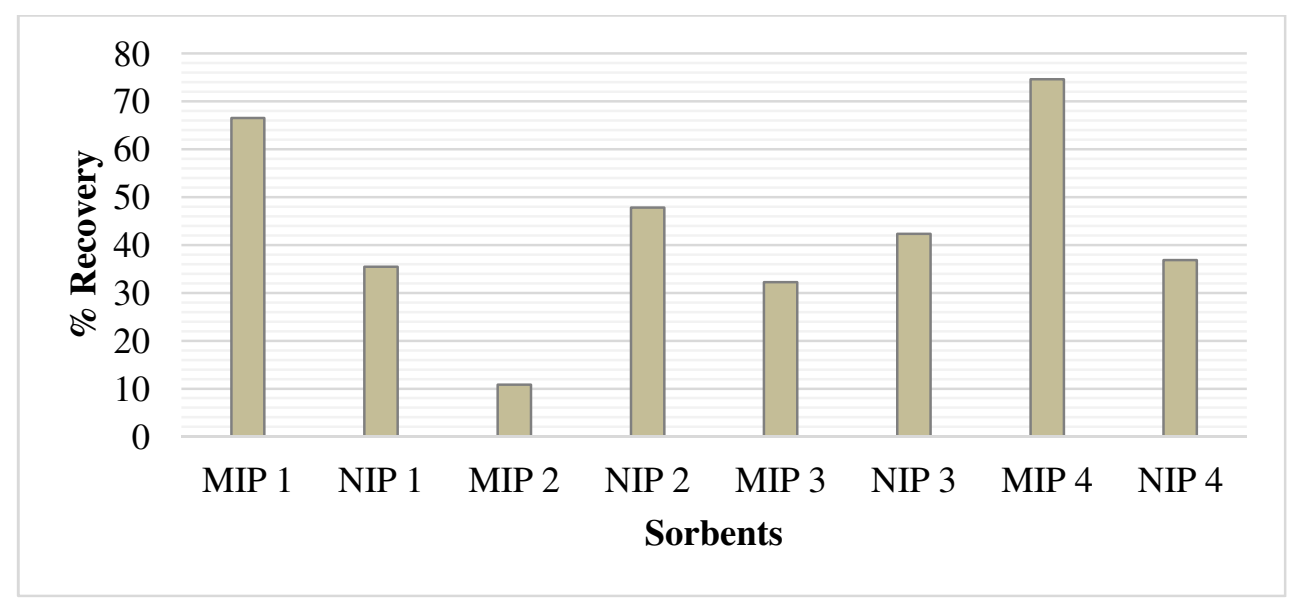

Fig.-5: Recovery Percentage of Atenolol in Serum Samples using MIP and NIP

\section{Physical Characterization}

Physical characterization tests are carried out to determine the physical form of the polymer before and after used as cartridges. SEM / EDS characterization only made to MIP 4/NIP 4 and MIP 1/NIP 1. This selection is based on the results of percent recovery, which is close to the requirements $(80 \%)$, namely $66.54 \%$ for MIP 1 and $74.64 \%$ for MIP $4{ }^{24}$.

The SEM results show that MIP 4 (Figure 6) has a larger cavity compared to the MIP 1 (Fig.-7), which allows for deposition MIP 4 to adsorb more analytes. This correlates with better recovery values for MIP 4 (close to $80 \%$ ).

Based on Fig.-7, the tested polymer did not experience significant changes. The polymer looks to have the same surface shape and the same cavity. So it can be concluded that polymers made with the bulk method have better physical form stability compared to polymers made by a depositional polymerization method. Pore size is an essential factor in determining the adsorption and release of analytes ${ }^{24,25}$. According to the International Union of Pure and Applied Chemistry classification, porous materials can be classified according to the size of their pores: including micropores (less than $2 \mathrm{~nm}$ ), mesopores $(2-50 \mathrm{~nm})$, macropores $(50-200 \mathrm{~nm} \text { ), and gigapore (more than } 200 \mathrm{~nm})^{24}$. Based on the test results, it can be seen that MIP 4 and NIP 4 polymers have a mesopore form, where the mesopore form is the best form for polymers.MIP 4 has better adsorption capability than NIP 4. This can be seen from the data on pore diameter, pore surface area,and pore volume. MIP $4(17.7927 \mathrm{~nm})$ has a small pore diameter compared to NIP $4(21.2076 \mathrm{~nm})$ which causes the surface area that is in contact with the analyte to be wider, thus causing the surface capacity for adsorption to be higher and the analyte more adsorbed on MIP 4 compared to NIP $4^{24}$.Type III isotherms confirm the mesopore structure of the polymer in Figures 8 . The type of isotherm, according to the IUPAC classification indicates that the polymer is composed of multilayers. This causes a lateral interaction between strongly adsorbed molecules compared to the interaction between the surface of the adsorbent and adsorbate, which shows both polymers both MIP 4 


\section{RASĀYAN J. Chem.}

Vol. 12 | No. 3 |1269 - 1278| July - September | 2019

and NIP 4 can absorb analytes (atenolol), so the results obtained do not show any significant differences between MIP 4 and NIP 4.
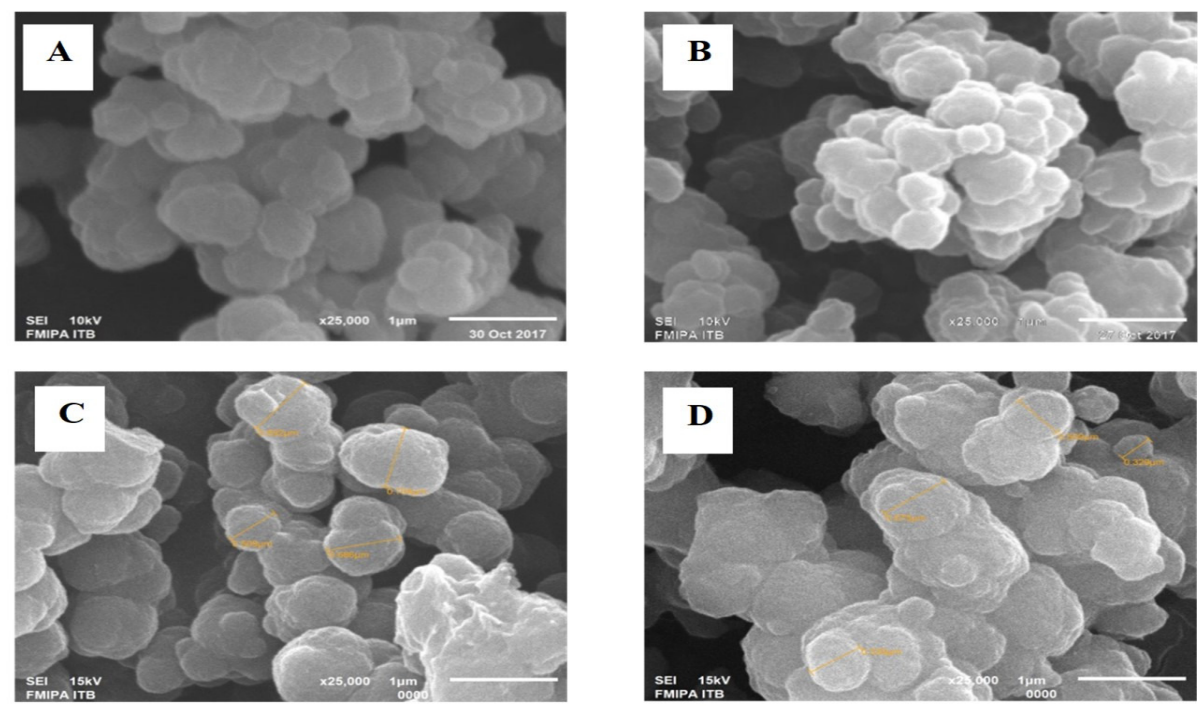

Fig.-6: 6MIP 4 and NIP 4 Polymer Morphology (a) MIP 4 before used as SPE Cartridge (b) NIP 4 before used as SPE Cartridge (c) MIP 4 after used as SPE Cartridge (d) NIP 4 after used as SPE Cartridge
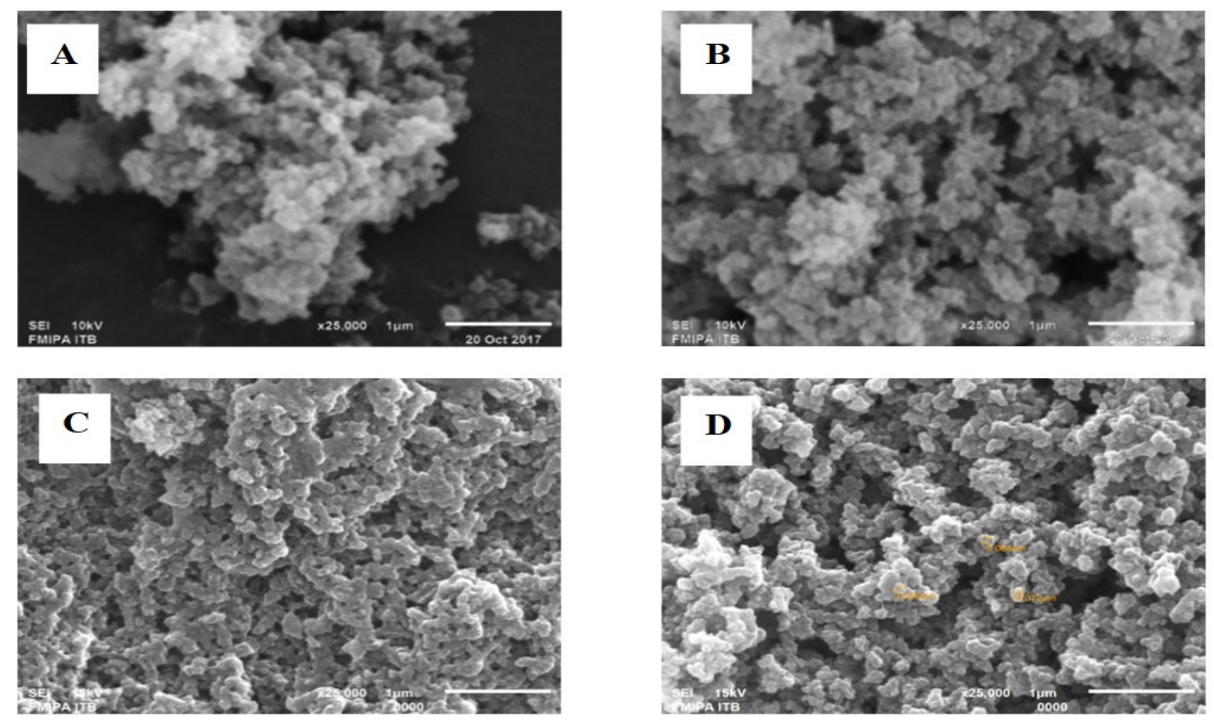

Fig.-7: MIP 1 and NIP 1 Polymer Morphology (a) MIP 1 before used as SPE Cartridge (b) NIP 1 before used as SPE Cartridge (c) MIP 1 after used as SPE Cartridge (d) NIP 1 after used as SPE Cartridge

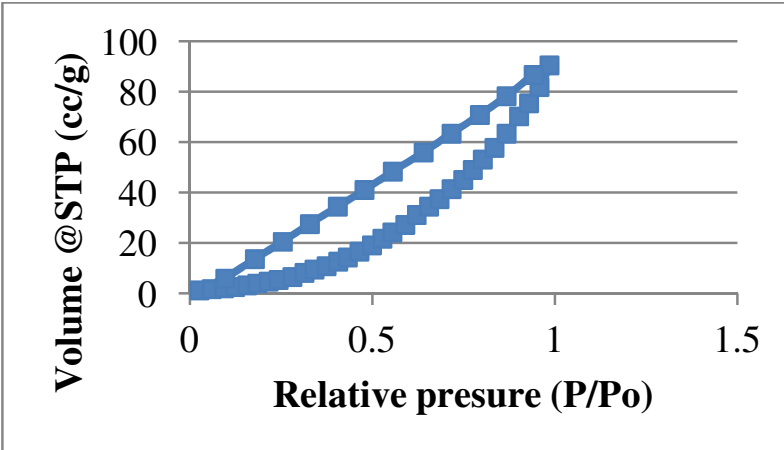

(a)

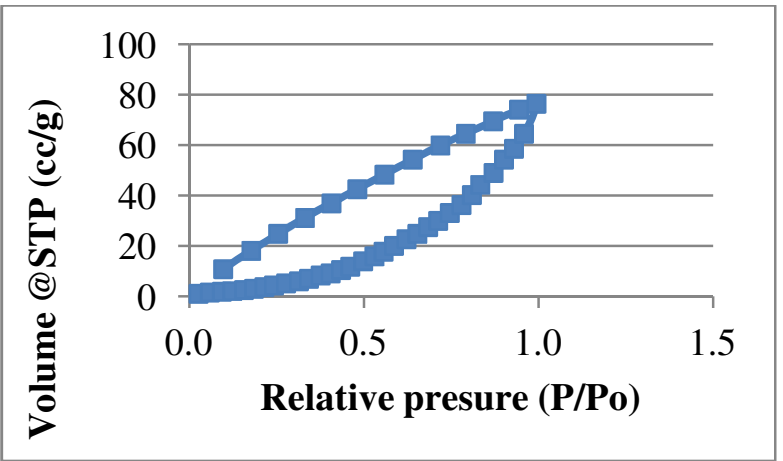

(b)

Fig.-8: Adsorption Isotherm Curve Precipitation (a) MIP 4 (b) NIP 4 
RASĀYAN J. Chem.

Vol. 12 | No. 3 |1269 - 1278| July - September | 2019

\section{CONCLUSION}

Four polymers optimized and applied to the serum were showed that the polymer made by the precipitation method in butanol solvents produced the best recovery value (74.64\%). The results of offline performance characterization show that the four sorbents can not be used as extracting materials because they have a percent recovery value that is less than the requirement $(>80 \%)$. The results of physical characterization show that there is no change in the functional group between sorbent before and after use and sorbent made by the method of bulk has better physical stability than sorbent made by the precipitation method.

\section{ACKNOWLEDGMENT}

The authors thank The Ministry of Research and Technology and Higher Education of the Republic of Indonesia for research funding trough Penelitian Terapan Unggulan Perguruan Tinggi (PTUPT) 2018 Grand No. 381c/UN6.O/LT/2018.

\section{REFERENCES}

1. E. Davis, R. Loiacono, R.J. Summers, Br. J. Pharmacol., 154, 584(2008), DOI: 10.1038/bjp.2008.164.

2. M.C. Hennio, J. Chromatogr. A, 856, 3(1999), DOI: 10.1016/S0021-9673(99)00832-8.

3. F.D.S. Leite, V.A. Pereira, C.E. Omosako, M.J.C. Carmona, J.O.C. Auler, S.R.C.J Santos, Therapeutic Drug Monitoring, 28, 237(2006), DOI: 10.1097/01.ftd.0000198647.39751.f5.

4. R.N. Goyal, V.K. Gupta, M. Oyama, N. Bachheti, Electrochem. Commun, 8, 65(2006), DOI: 10.1016/j.elecom.2005.10.011.

5. M.A. El Ries, Anal.Lett., 28, 1629(1995), DOI: 10.1080/00032719508002770

6. R. Afonso, A.P.P. Eisele, J.A. Serafim, A.C. Lucilha, E.H. Duarte, C.R.T. Tarley, E.R. Sartori, L.H. Dall'Antonia, J. Electroanal. Chem., 765, 30(2016), DOI:10.1016/j.jelechem.2015.10.014.

7. B. Yilmaz and S. Arslan. J. Chromatogr Sci, 49, 365(2011), DOI: 10.1093/chromsci/49.5.365.

8. K. Golker, B.C.G. Karlsson, G.D. Olsson, A.M. Rosengren, I.A Nicholls, Macromolecules, 46, 1408(2013), DOI: 10.1021/ma3024238.

9. M. Bompart, L.A. Gheber, Y. De Wilde, and K. Haupt, Biosens. Bioelectron, 25, 568(2009), DOI: $10.1016 /$ j.bios.2009.01.020.

10. S.A. Zaidi, S.M. Lee, W.J. Cheong, J. Chromatogr. A., 1218, 1291(2011) DOI: $10.1016 /$ j.chroma.2010.12.117.

11. K. Golker, B.G.C. Karlsson, G.D. Olsson, A.M. Rosengren, I.A. Nicholls, Macromolecules, 46, 1408(2013), DOI: 10.1021/ma3024238.

12. M. Bompart, L.A. Gheber, Y. De Wilde, K. Haupt, Biosens. Bioelectron., 25, 568(2009), DOI: $10.1016 /$ j.bios.2009.01.020.

13. S.A. Zaidi, S.M. Lee, W.J. Cheong, J. Chromatogr. A. 1218, 1291(2011), DOI: $10.1016 /$ j.chroma.2010.12.117.

14. H.A. Andreetta and L. Bruzzone, Anal. Lett, 41(1), 36(2008), DOI: 10.1080/00032710701748465.

15. A.N. Hasanah, S. Suryana, Mutakin, D. Rahayu, Asian J. Chem., 29(11), 2429(2017), DOI: 10.14233/ajchem.2017.20728.

16. G. Vasapollo, R.D. Sole, L. Mergola, M.R. Lazzoi, A. Scardino, S. Scorrano, G. Mele, Int. J. Mol. Sci., 12, 5908(2011), DOI:10.3390/ijms12095908.

17. P. Thordarson, Chem. Soc. Rev, 40, 1305(2011), DOI: 10.1039/C0CS00062K

18. A.N. Hasanah, T.N.D. Utari, R. Pratiwi, J. Anal. Method Chem., 1(2019), DOI: $10.1155 / 2019 / 9853620$.

19. L. Chen, S. Xu, J. Li, RSC Adv.. 40, 2922(2011), DOI: 10.1039/C0CS00084A.

20. T. Nurhayati, I. Yanti, Royani, Widayani, Khairurrijal. Journal of Physics : Conference Series, 739(2016), DOI:10.1088/1742-6596/739/1/012143.

21. H. Yan, and H.R. Kyung. Int. J. Mol. Sci, 7, 155(2006), DOI:10.3390/i7050155.

22. T. Beyki and M.J. Asodallahzadeh, J. Water Environ. Nanotechnol, 1(1), 19(2016), DOI: $10.7508 / J W E N T .2016 .01 .003$.

23. R.J. Umpleby II, S. Baxter, M. Bode, J.K. Berch, R.N. Shah, K.D. Shimizu, J Chromatogr B Analyt Technol Biomed Life Sci., 804, 141(2004), DOI:10.1016/s0003-2670(00)01211-3. 


\section{RASĀYAN J. Chem.}

Vol. 12 | No. 3 |1269 - 1278| July - September | 2019

24. S.A. Wei, B. Molinelli, Mizaikoff, Biosens. Bioelectron., 21, 1943(2006), DOI: 10.1016/j.bios.2005.09.017.

25. https://pubchem.ncbi.nlm.nih.gov/compound/4093

26. http://www.fda.gov/downloads/Drugs/GuidanceComplianceRegulatoryInformation/Guidances/UCM0 70107.pdf

27. Y. Cai, Int. J. Nanomed., 8, 1111(2013), DOI: 10.2147/IJN.S41271.

28. M.C. Djuanidi and Y. Astuti, Rasayan J. Chem., 12(2), 809(2019), DOI: 10.31788/RJC.2019.1225120.

[RJC-5202/2019] 